\title{
Analysis of Singularities From Modulus Maxima of Complex Wavelets
}

\author{
Chun-Liang Tu, Wen-Liang Hwang, Member, IEEE, and Jinn Ho
}

\begin{abstract}
Complex-valued wavelets are normally used to measure instantaneous frequencies, while real wavelets are normally used to detect singularities. We prove that the wavelet modulus maxima with a complex-valued wavelet can detect and characterize singularities. This is an extension of the previous wavelet work of Mallat and Hwang on modulus maxima using a real wavelet. With this extension, we can simultaneously detect instantaneous frequencies and singularities from the wavelet modulus maxima of a complex-valued wavelet. Some results of singularity detection with the modulus maxima from a real wavelet and an analytic complex-valued wavelet are compared. We also demonstrate that singularity detection methods can be employed to detect the corners of a planar object.
\end{abstract}

Index Terms-Continuous wavelet transform, corner detection, singularity, wavelet modulus maxima.

\section{INTRODUCTION}

I $\mathrm{N}$ recent years, singularity analysis has emerged as a multiple-area problem solving method [2], [3], [6], [19], [13], [12]. One pioneering work [15] studied the detection of the singularities of local homogeneous functions from the phase of wavelet transforms. Singularities of a signal can be characterized by the modulus of their wavelet transforms [10], [17]. Furthermore, it was shown in [14] that only from the modulus maxima of a real-valued wavelet can all the singularities be detected and characterized. However, [14] focused on the modulus maxima of real wavelets for singularity detection and characterization, not on the modulus maxima of complex-valued wavelets.

A complex-valued analytic wavelet only responds to the nonnegative frequencies of a given signal and, thus, produces a transform whose moduli are less oscillatory than in the case of a real wavelet. Analytic wavelets have been widely used to detect and characterize the instantaneous frequencies of signals and images based on ridges [7], [22], which mark the places in the time-frequency plane where most of the local energies of a signal are concentrated. One can detect ridges from either the phase or the magnitude of a wavelet transform [5]. Instantaneous frequencies can be approximately identified from the modulus maxima of a real wavelet based on general maxima, which are the locations of the largest modulus along maxima lines [14].

Manuscript received May 25, 2002; revised October 1, 2004.

C.-L. Tu was with the Computer and Communications Research Laboratories, ITRI, Taiwan, R.O.C. He is now with Aiptek Inc., Hsinchu, Taiwan, R.O.C. (e-mail: JohnTu@aiptec.com.tw).

W.-L. Hwang and J. Ho are with the Institute of Information Science, Academia Sinica, Nankang, Taipei, Taiwan, R.O.C. (e-mail: whwang@iis. sinica.edu.tw; hjinn@iis.sinica.edu.tw).

Communicated by G. Battail, Associate Editor at Large.

Digital Object Identifier 10.1109/TIT.2004.842706
However, compared to ridges, general maxima are not as mathematically or practically elegant for detecting and characterizing instantaneous frequencies.

Our main contribution is to prove that the modulus maxima of the wavelet transform using a complex-valued wavelet can detect and characterize singularities. Because of this, we are able to simultaneously process instantaneous frequencies and singularities from the modulus maxima of the wavelet transform using a complex-valued wavelet. By simulation, we demonstrate that using the modulus maxima of a complex-valued analytic wavelet is better for detecting and estimating singularities than using a real wavelet when a signal is embedded in an additive white Gaussian noise environment. Additionally, we apply a singularity method to detect corners from the contour of a planar object. Through simulation, we show that complex-valued analytic wavelets outperform real wavelets in this task.

The remainder of this paper is organized as follows. Section II contains background material on the complex-valued wavelet transform. In Section III, we review the results of singularity processing based on the modulus maxima of a real wavelet. We also present our results based on a complex-valued wavelet and demonstrate its performance by comparison with singularity detection and estimation. In Section IV, we show that a singularity method can be applied to detect corner points. In addition, we demonstrate and compare examples with a real wavelet and a complex-valued wavelet. Finally, in Section V, we present our conclusion.

\section{COMPLEX-VALUED WAVELETS}

The continuous wavelet transform was first introduced by Morlet and Grossmann [9]. To review, let $\Psi(x)$ be a complexvalued function, which is said to be a wavelet if its integral is zero

$$
\int_{-\infty}^{\infty} \Psi(x) d x=0 .
$$

The continuous wavelet transform of a function $f(x) \in \mathcal{L}^{2}(R)$ with respect to the wavelet $\Psi(x)$ is defined as

$$
\mathcal{W} f(s, x)=\int_{-\infty}^{\infty} f(a) \bar{\Psi}_{s}(a-x) d a
$$

where $\Psi_{s}(x)=\frac{1}{s} \Psi\left(\frac{x}{s}\right)$ and $\bar{\Psi}_{s}(x)$ denotes the complex conjugate of $\Psi_{s}(x)$. A wavelet $\Psi(x)$ is said to have $n$ vanishing moments if and only if for all positive integers $k<n$, it satisfies

$$
\int_{-\infty}^{+\infty} x^{k} \Psi(x) d x=0
$$


The wavelet transform is invertible, and $f(x)$ is recovered with

$$
f(x)=2 \mathcal{R}\left\{\frac{1}{C_{\Psi}} \int_{0^{+}}^{+\infty} \int_{-\infty}^{+\infty} \mathcal{W} f(s, u) \Psi_{s}(u-x) d u \frac{d s}{s}\right\}
$$

where

$$
C_{\Psi}=\int_{0}^{\infty}|\hat{\Psi}(w)|^{2} \frac{d w}{w}
$$

and $\hat{\Psi}(w)$ is the Fourier transform of $\Psi(x)$.

Several interesting complex-valued wavelets have been used in a wide variety of applications. The Cauchy wavelet

$$
\Psi(x)=\frac{1}{2 \pi} \frac{1}{(1-i x)^{2}}
$$

has been used in quantum mechanics [1], while the Morlet wavelet

$$
\Psi(x)=e^{-\frac{x^{2}}{2 \sigma^{2}}} e^{i \omega_{0} x}
$$

has been used to detect instantaneous frequencies and analyze textured images [7], [11]. Strictly speaking, the Morlet wavelet is not a true wavelet in that its integral is not zero. However, for a large enough $\omega_{0}$ (in practice, larger than 5), the integral of $\Psi(x)$ is small enough to ensure that it can be used numerically, as if it were a wavelet. A popular wavelet in computer vision is the $n$th derivative of the Gaussian function

$$
\psi_{n}(x)=-\frac{d^{n}}{d x^{n}} e^{-\frac{x^{2}}{2}} .
$$

These wavelets can be easily turned into analytic wavelets by canceling their negative frequencies by means of the Hilbert transform $\mathcal{H}$, i.e., by considering $\Psi_{n}(x)=(1+i \mathcal{H}) \psi_{n}(x)$. The frequency response is then

$$
\hat{\Psi}_{n}(\xi)=K_{n} \xi^{n} e^{-\frac{\xi^{2}}{2}} \chi_{(0, \infty)}(\xi)
$$

where $\chi_{(0, \infty)}(\xi)$ denotes the Heaviside step function, which is equal to 1 when $\xi>0$ and to 0 otherwise. $K_{n}$ denotes a normalization constant. The parameter $n$ gives different numbers of vanishing moments of wavelets. When performing wavelet singularity analysis, the number of vanishing moments is very important, as it provides an upper bound measurement for singularity characterization.

\section{Singularity Detection From COMPLEX-VALUED WAVELETS}

The Lipschitz exponent is a measurement of the strength of a singularity. It has been shown that the Lipschitz exponent of a local singularity can be characterized by wavelet transforms. One can find related results in [10], [17], [18]. Furthermore, Mallat and Hwang [14] showed that the Lipschitz exponent can also be computed with the restriction of the wavelet transforms $\mathcal{W} f(s, x)$, placed on their local modulus maxima at each scale $s$. However, their results are valid only if the wavelet is real.

In this section, we first review the results in [14] regarding detection and characterization from the modulus maxima of a real wavelet. We then extend part of these results, using the modulus maxima of a complex-valued wavelet, to show that the modulus maxima of a complex-valued wavelet can also be used to detect and characterize singularities. We begin by introducing some related definitions.

\section{Definitions:}

- If a function $f(x)$ satisfies $|f(x)-f(y)| \leq c|x-y|^{\alpha}$ with $c>0, \alpha \in[0,1]$ and $x, y \in(a, b)$, we say that the function $f(x)$ is uniformly Lipschitz of exponent $\alpha$ over the interval.

- Let $n$ be a positive integer, and $n \leq \alpha \leq n+1$. A function $f(x)$ is said to be uniformly Lipschitz of exponent $\alpha$ over the interval $(a, b)$ if for any $x$ in the interval there exists a polynomial $P_{n}$ of order $n$ and a constant $C$ such that $\left|f(x)-P_{n}(x)\right| \leq C|h|^{\alpha}$ for $h$ small enough.

- We say that the Lipschitz (regularity) of $f(x)$ at $x_{0}$ is $\alpha$ if $\alpha$ is a superior bound of all Lipschitz exponents of $f(x)$ at $x_{0}$. A function is singular at $x_{0}$ if it does not have Lipschitz regularity 1 at $x_{0}$.

- In numerical computations, we may encounter negative Lipschitz exponents. The negative Lipschitz exponents for tempered distributions are defined in [14] as follows: The distribution $f(x)$ is said to be uniformly Lipschitz $\alpha$ over interval $(a, b)$, if and only if its primitive is uniformly Lipschitz $\alpha+1$ in the interval.

- A modulus maximum is the point $\left(s_{0}, x_{0}\right)$ where

$$
\left|\mathcal{W} f\left(s_{0}, x\right)\right|<\left|\mathcal{W} f\left(s_{0}, x_{0}\right)\right|
$$

when $x$ belongs to either the right or the left neighborhood of $x_{0}$, and

$$
\left|\mathcal{W} f\left(s_{0}, x\right)\right| \leq\left|\mathcal{W} f\left(s_{0}, x_{0}\right)\right|
$$

when $x$ belongs to the opposite neighborhood of $x_{0}$.

- A maxima line is a connected curve of the modulus maxima in the scale space $(s, x)$.

\section{A. Isolated Singularity}

The theorems given in [14] state that the wavelet modulus maxima of a real wavelet can detect all singular points in a given interval. Maxima lines converge as the scale $s$ decreases to (while not being limited to) all the singular points in the interval. From the values of the lines, one can characterize their Lipschitz regularities.

In order to extend the above-mentioned results from a real wavelet to a complex-valued wavelet, one should place constraints on the real and imaginary parts of wavelet transforms. For convenience, we introduce the following definition: two functions $a(x)$ and $b(x)$ are finite deviations within a given interval $I$ if for any subinterval (of length $\epsilon$ ) of $I$, they can be divided into at most $M(\epsilon)$ intervals $v_{1}, v_{2}, \ldots, v_{M}$, where in each interval $v_{i}$ is either

- (Type I) $a(x)=b(x)$ for all $x$ in $v_{i}$ or

- (Type II) $a(x)=b(x)$ at the two ends of the interval, but $a(x) \neq b(x)$ for any $x$ in between them.

Two functions are not finite deviations within an interval only if they have an indefinite number of intersections (of Type I, or 


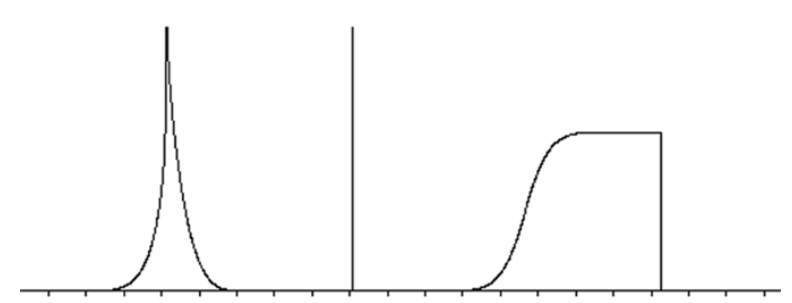

(a)

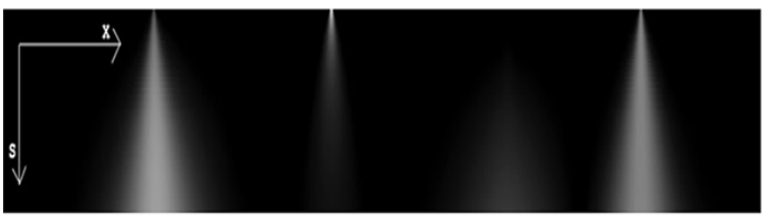

(b)
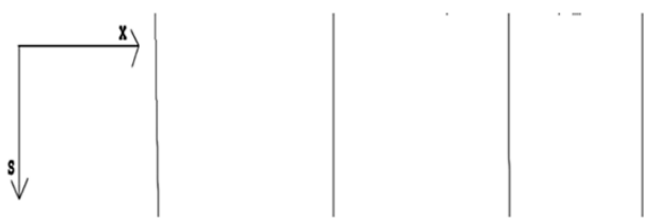

(c)

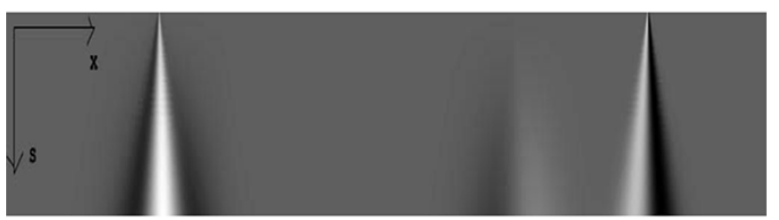

(d)
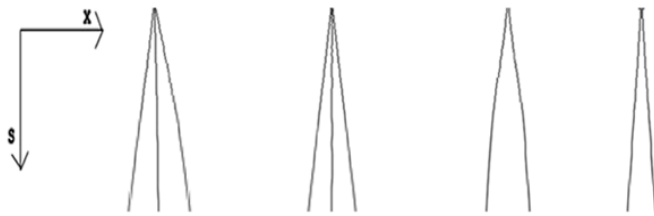

(e)

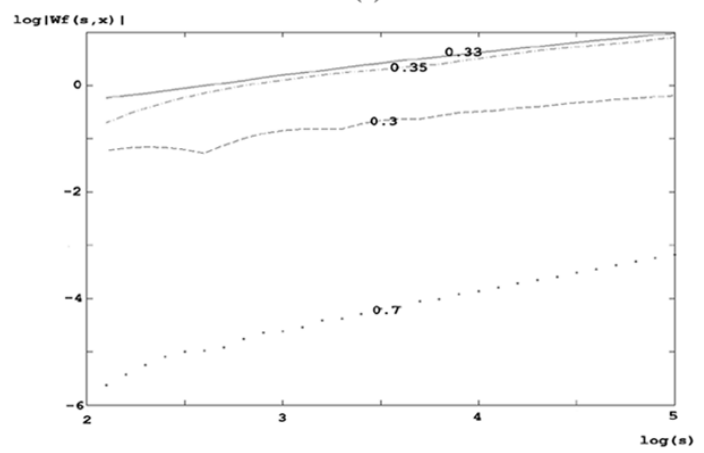

(f)

Fig. 1. (a) There are four singularities that follow from left to right in this signal. In the left neighborhood of the first singularity, it locally behaves like $O\left(|x|^{0.2}\right.$ ), whereas in its right neighborhood, it behaves like $O\left(|x|^{0.6}\right)$. The second singularity is a Dirac whose Lipschitz is -1 . The Lipschitz exponent of the third singularity is 1.5. The fourth is a step singularity. (b) The modulus of the complex-valued wavelet transform of (a). (c) The maxima line of (b). (d) The real part of the wavelet transform in (b). (e) The maxima lines of (d) in which there are three maxima lines corresponding to the first singularity. The left maxima line corresponds to the left neighborhood of the singularity with $O\left(|x|^{0.2}\right)$, and the right maxima line corresponds to the right neighborhood of the singularity with $O\left(|x|^{0.6}\right)$. The middle maxima line is the compromise behavior between the left and right neighborhoods of the singularity. (f) Decay of $\log (|\mathcal{W} f(s, x)|)$ as a function of $\log (s)$ along the maxima lines of the first singularity. The maxima line of (c) is plotted with a solid line. The three maxima lines of (e), from left to right, are plotted with a long dash line "-, " short dash line "-," and a dotted line ".". Their slopes are recorded on the lines.

Type II, or both) in the interval. An example of two functions, which are not finite deviations for any interval including $x=0$, are $\cos \left(\frac{1}{x}\right)$ and $\sin \left(\frac{1}{x}\right)$. The two functions have an unbounded number of intersections for any subinterval around $x=0$.
We show in the following theorem that if there are no complex-valued wavelet modulus maxima in all sufficiently small scales in a given interval, and if the real and imaginary parts of the wavelet transform in all these scales are finite-deviations, 
then the function is uniformly Lipschitz $\alpha$ for any $\alpha<n$, in this interval.

Theorem 1: Let $n$ be a strictly positive integer. Let $\Psi(x)$ be a complex-valued wavelet that has compact support, has $n$ vanishing moments, and is $n$ times continuously differentiable. Let $f(x) \in L^{1}([a, b])$.

- If a scale $s_{0}>0$ exists such that for all scales $s<s_{0}$ and $x \in(a, b),|\mathcal{W} f(s, x)|$ has no local maxima, and if the $n$th $(n>0)$ derivatives of $\operatorname{Re}(\mathcal{W} f(s, x))$ and $\operatorname{Im}(\mathcal{W} f(s, x))$ are finite deviations for each scale $s$, then for any $\epsilon>0$ and $\alpha<n, f(x)$ is uniformly Lipschitz $\alpha$ in $(a+\epsilon, b-\epsilon)$.

- If $\Psi(x)$ is the $n$th derivative of a smoothing function, then $f(x)$ is uniformly Lipschitz $n$ on any such interval $(a+$ $\epsilon, b-\epsilon)$.

The proof of this theorem is given in Appendix I. The theorem indicates that if the real and imaginary parts of the wavelet transform for a function $f(x)$ are finite deviations for all sufficiently small scales, then from the wavelet modulus maxima of a complex-valued wavelet $\Psi(x)=\psi_{R}(x)+i \psi_{I}(x)$, we can detect all the singular points of the function $f(x)$, as is the case with the real wavelet $\psi_{R}(x)$. The following theorem states that the wavelet modulus maxima of a complex-valued wavelet can characterize singularities.

Theorem 2: Let $x_{0} \in(a, b)$ and let the $n$th $(n>0)$ derivative of $\operatorname{Re}(\mathcal{W} f(s, x))$ and $\operatorname{Im}(\mathcal{W} f(s, x))$ be finite-deviations for each scale $s$. If a scale $s_{0}>0$ and constants $C$ and $A$ exist such that for $x \in(a, b)$ and $s<s_{0}$, all the modulus maxima of $\mathcal{W} f(s, x)$ belong to a cone defined by

$$
\left|x-x_{0}\right| \leq C s
$$

such that, at each modulus maxima $(s, x)$ in the cone

$$
|\mathcal{W} f(s, x)| \leq A s^{\alpha} ;
$$

then $f(x)$ is uniformly Lipschitz $n$ at $x$ when $x \neq x_{0}$, and $f(x)$ is Lipschitz $\alpha$ at $x_{0}$ when $\alpha<n$ is a noninteger.

The proof of this theorem is similar to that given in [14, Appendix I]. We further illustrate it in Appendix II in this paper. In order to apply our theorems, the extra finite-deviations condition for the real and complex parts of the wavelet transform and those required in [14] are needed. If the real and imaginary parts of the wavelet transform of $f(x)$ are not finite deviations within an interval, then they must have an indefinite number of intersections (of Type I, or Type II, or both). Hence, there is an indefinite number of oscillations of $f(x)$ within the interval. In many applications, however, signals do not oscillate indefinitely over an interval.

In order to obtain the Lipschitz exponent numerically, (7) is usually rewritten as

$$
\log |\mathcal{W} f(s, x)| \leq \log (A)+\alpha \log (s) .
$$

In [14], $A$ and $\alpha$ are computed by

$$
\min _{A, \alpha}\left(\sum_{s}(\log |\mathcal{W} f(s, x)|-\log (A)-\alpha \log (s))^{2}\right) .
$$

In practice, however, it is known that estimating $\alpha$ by means of least squared fitting is not reliable. A more robust least median of squares regression [21] can be used to find $A^{*}$ and $\alpha^{*}$ that minimize the objective function

$$
f(A, \alpha)=\operatorname{median}_{s}\left((\log |\mathcal{W} f(s, x)|-\log (A)-\alpha \log (s))^{2}\right) .
$$

Fig. 1 shows the wavelet transforms and the wavelet modulus maxima of a function obtained by using the wavelet $(1+$ $i \mathcal{H}) \psi(x)$, where $\psi(x)$ is the second derivative of a Gaussian function. Our function is constructed with the isolated singularities shown in Fig. 1(a). There are four maxima lines in Fig. 1(c): the first line from the left is not vertical because the two sides of the corresponding singular point behave differently, while the rest of the maxima lines are vertical. More maxima lines converge to each singular point in Fig. 1(e) than in Fig. 1(c) due to the oscillation in the modulus of the real wavelet transform. In Fig. 1(e), three maxima lines correspond to the first singular point at the left under the real part of the wavelet transform, while only one maxima line corresponds to this singular point under the complex-valued wavelet transform. In Appendix IV, we calculate the modulus maxima of this singularity with real and analytical wavelets.

We compare the detection and characterization efficacies of real and analytic wavelets for the singularities in Fig. 1 by adding white Gaussian noises to the signal such that the signal-to-noise ratios (SNRs) of the resultant signals vary from 40 to $10 \mathrm{~dB}$. We use the algorithm proposed in [14] to generate maxima lines from the modulus maxima of wavelet transforms. The location of a singularity is measured by the mean of the positions in the finest scale from the maxima lines corresponding to the singularity. There is more than one maxima line for a singularity if a real wavelet is used; thus, the estimated location of the singularity is the mean of the location of each maxima line. The left column of Fig. 2 compares the statistics of location detection for each singularity, while the right column of the figure compares the statistics of characterization of each singularity by measuring its Lipschitz exponent. Note that the mean of the measured Lipschitz exponents of each maxima line is used for the real wavelet, except for the first singularity on the left. The Lipschitz exponent of this singularity for the real wavelet is measured from the modulus of the middle maxima line. The dashed line in each part of the figure indicates the value if a clean signal has been analyzed. The third singularity has a Lipschitz exponent of 1.5 , indicating that the value of a modulus maxima in a finer scale is less than that in a coarser scale. For this singularity, the noise variance in the finer scale dominates the wavelet modulus of the singularity; thus, its correct maxima lines are not discernible. However, the analytic wavelet gives a moderate error of up to $30 \mathrm{~dB}$ in Lipschitz exponent estimation, while the real wavelet looses the value, even at $40 \mathrm{~dB}$. The bottom right part of the figure shows that the analytic wavelet yields a better estimation of the step edge.

Remark: The resolution of a discrete signal is limited to, say, $s_{\text {small }}$. Thus, when estimating the Lipschitz exponent of a singular point in the signal, we know that the scale $s$ cannot be arbitrarily small, i.e., it is constrained by $s \geq s_{\text {small }}$. The Lipschitz exponents estimated in Fig. 1 are the best possible solutions when applying our method with $s \geq s_{\text {small }}$. 

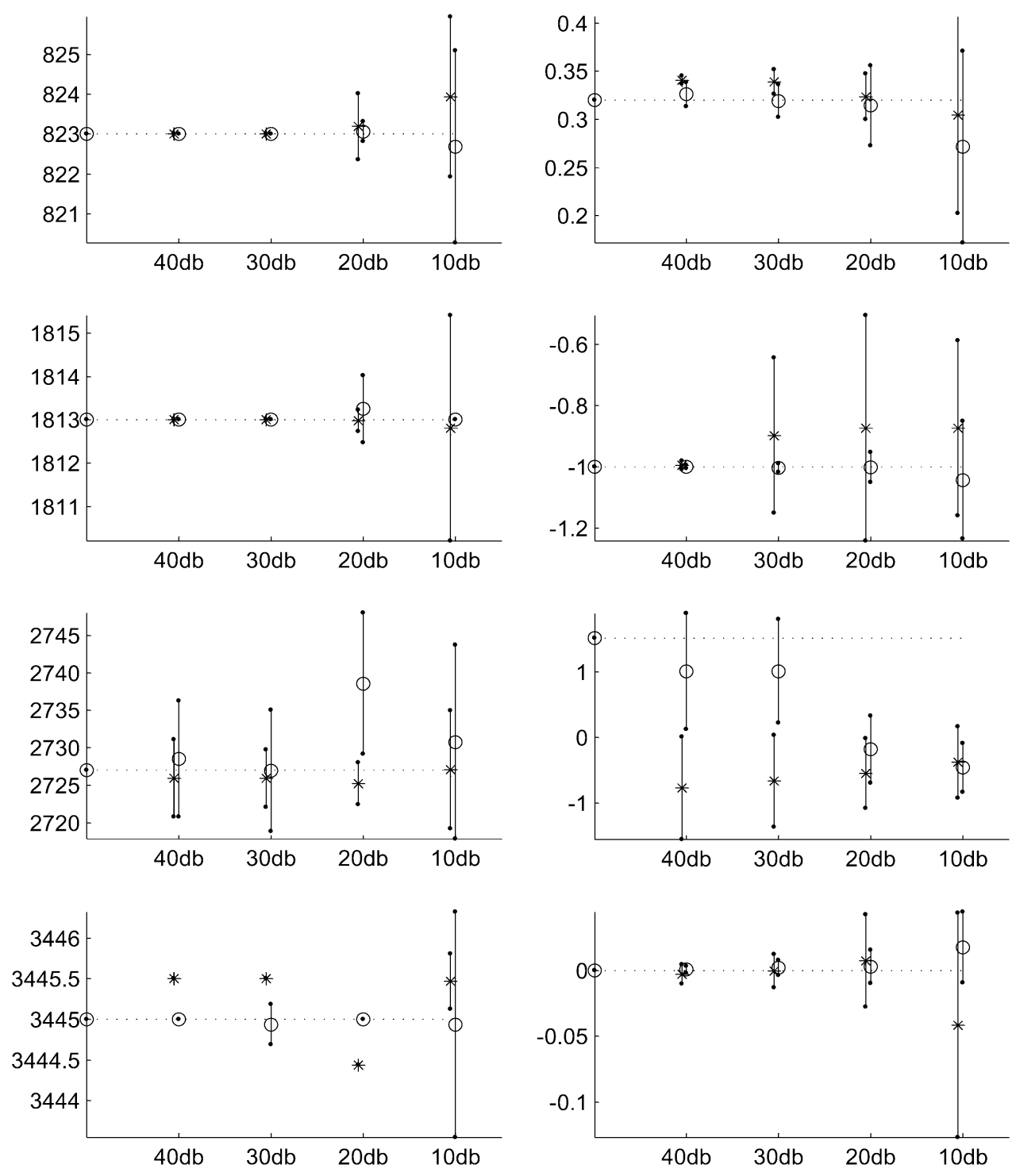

Fig. 2. Comparison of singularity detection and characterization of the signal in Fig. 1(a) using a real and an analytic wavelet in additive white Gaussian noise environments. The SNRs vary from 40 to $10 \mathrm{~dB}$. The results of the real wavelet are indicated by $*$ and that of analytic wavelet by o. The left column shows the mean and standard deviation of the location estimation of each singularity, while the right column shows those of Lipschitz exponent estimation. Each statistic was measured by 16 experiments. The horizontal dashed lines give the values measured in the clean signal. The subfigures from top to bottom correspond to the singularities from the left to right.

\section{Corner Detection of Planar Shape Sketches}

The shape of a planar object has been widely used in computer vision to recognize and represent an object. The performance of a shape-recognition system depends heavily on the effectiveness of low-level processing of contour signals. Corner points, which are the locations on a contour where the curvature changes sharply, are regarded as the most descriptive features. Imaging a planar object usually produces quantization noise. In Fig. 3, the $\left\{B_{i}\right\}$ sequence is the contour of the original planar object, and the $\left\{A_{i}\right\}$ sequence is the contour of its image. Note that due to quantization to a lattice point, the two contours are different.

The contour of a planar object is usually represented as a onedimensional signal using the chain code method [4], [8]. We use $\theta(x)$ for the chain code, where $\theta(x)$ is the angle between the tangent $\boldsymbol{t}$ to the boundary of an object with arc length $x$ and the direction of the horizontal axis

$$
\theta(x)=\angle(\boldsymbol{t}(x), \text { horizontal axis }) .
$$

Let $\theta_{B}(x)$ and $\theta_{A}(x)$ be, respectively, the chain code representation of the planar object and its image; the difference between them is the quantization noise due to the digitizing process. The corner detection problem is to estimate the corners in the chain code $\theta_{B}(x)$ of the original planar object from the given chain code $\theta_{A}(x)$ of the imaged object.

Since two neighboring points of a digitized object have a limited number of directions, the chain code representation of the object's contour has a limited angle. If the direction of each segment is coded by using an 8-connectivity numbering scheme, such as the one shown in Fig. 4(a), then the chain code of an 


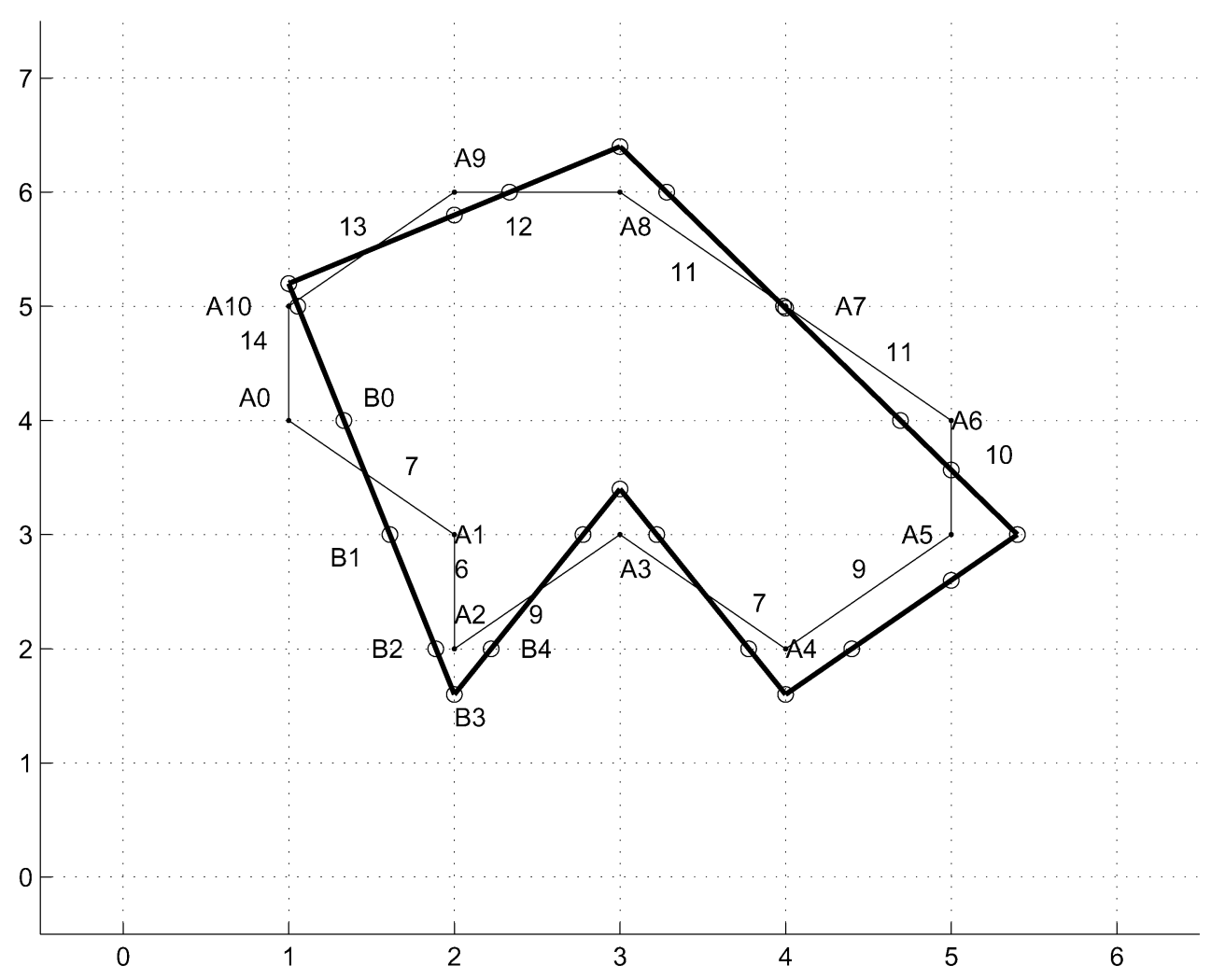

Fig. 3. Imaging a planar object. Note that $B_{2}, B_{3}, B_{4}$ in the contour of the original object are mapped to $A_{2}$ in the imaged object, $B_{1}$ to $A_{1}$, and $B_{0}$ to $A_{0}$, respectively. The number beside the segment is the chain code of the segment.

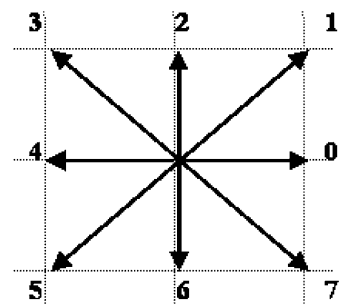

(a)

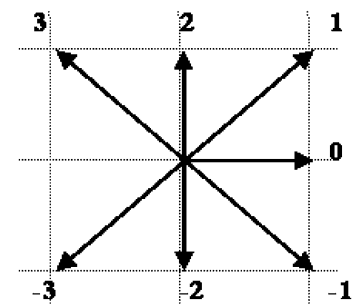

(b)

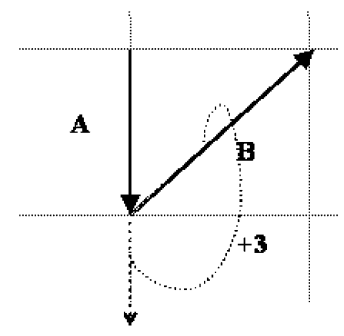

(c)

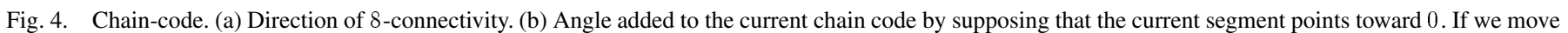
counterclosewise to the next segment by $\frac{\pi}{2}$, then the index of the angle to be added to our chain code is 2 . (c) An example.

imaged object can only have values in $(8 \times k+l) \frac{\pi}{4}$, where $k$ and $l$ are integers and $l$ is within 0 to 7 . In chain code implementation, an angle $\frac{i \pi}{4}$, where $i \in\{-3,-2,-1,0,1,2,3\}$ corresponds to a direction shown in Fig. 4(b), is added to the current chain code when we trace the contour to the next segment. For example, if the next segment has the same direction as the current segment, we add 0 to the current chain code. This implementation adds to our current chain code the smallest angle with range $(-\pi, \pi)$ between the current and the next segments. Fig. 4 (c) illustrates an example in which the angle $\frac{3 \pi}{4}$ is added to a chain code by moving counterclosewise from segment $A$ to segment $B$. The number beside each segment indicates the chain code of the segment after dividing it by $\frac{\pi}{4}$. For convenience, we hereafter represent the chain code by its number.

The derivative of the chain code of a planar object at $x$ is the curvature at that location. A corner point is a location where the curvature changes sharply, and the tangent to the contour is discontinuous. In [3], the chain code of a corner at $x=0$ is modeled as

$$
\theta(x)= \begin{cases}k_{1} x+c, & \text { if } x<0 \\ k_{2} x+c+\phi, & \text { if } x>0\end{cases}
$$

where $k_{1}, k_{2}$ are curvatures, $c$ and $\phi$ are, respectively, the angle and the angle discontinuity at $x=0$. By employing the wavelet transform with a wavelet that has at least two vanishing moments, we obtain $\mathcal{W}_{s} \theta(x)=0$ when $x \neq 0$ and

$$
\begin{aligned}
\mathcal{W}_{s} \theta(x)= & \int_{-\infty}^{0}\left(k_{1} a+c\right) \frac{1}{s} \Psi_{n}\left(\frac{x-a}{s}\right) d a \\
& +\int_{0}^{+\infty}\left(k_{2} a+c+\phi\right) \frac{1}{s} \Psi_{n}\left(\frac{x-a}{s}\right) d a \\
= & \phi \int_{0}^{+\infty} \frac{1}{s} \Psi_{n}\left(\frac{x-a}{s}\right) d a \\
& +\int_{-\infty}^{0}\left(k_{1}-k_{2}\right) a \frac{1}{s} \Psi_{n}\left(\frac{x-a}{s}\right) d a .
\end{aligned}
$$




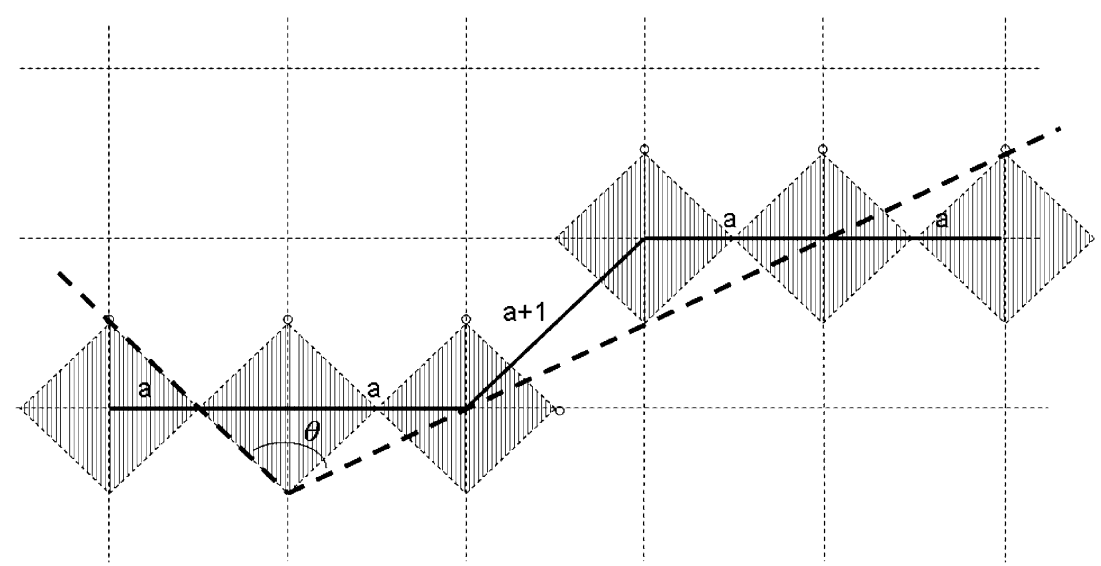

(a)

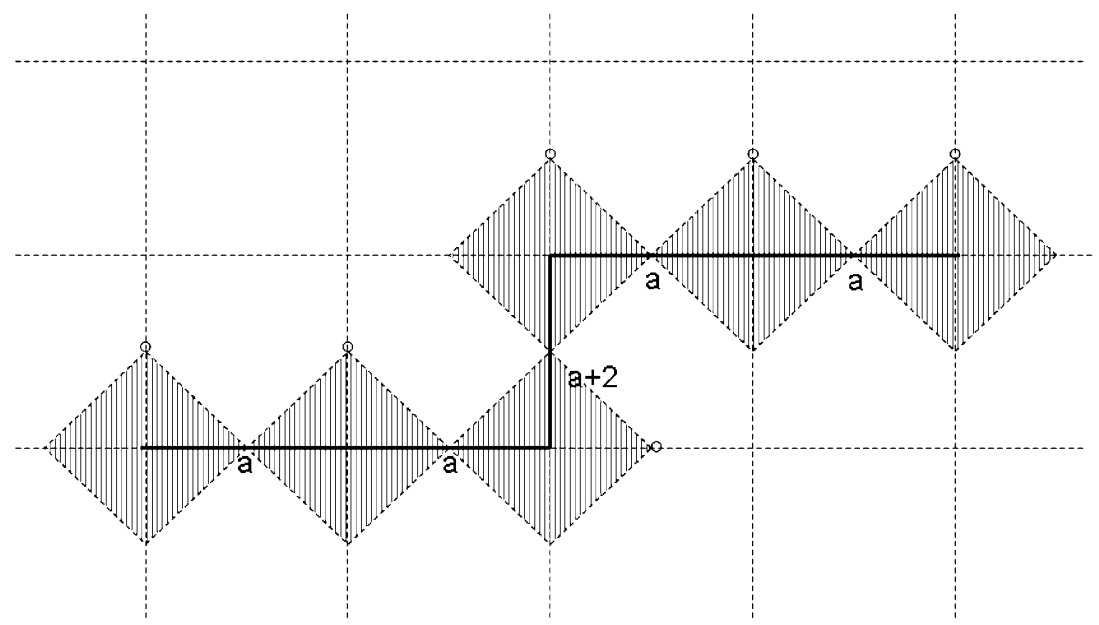

(b)

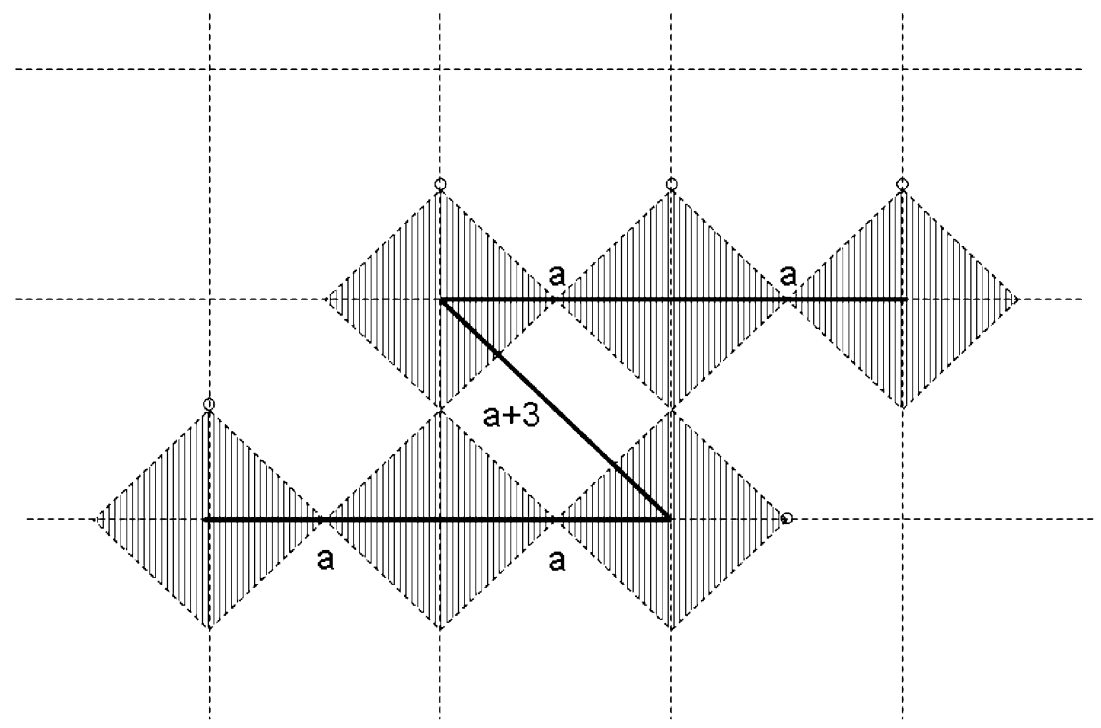

(c)

Fig. 5. A few patterns yield a chain code $(a, a, a+d, a, a)$, with $d \in\{ \pm 1, \pm 2, \pm 3\}$. We draw patterns of positive $d$. Patterns with negative $d$ values are similar and we, therefore, omit them. Any corner of a planar object, whose chain code is one of the patterns (as shown in solid line segments), cannot be detected by our method. The chain codes are a five-point Dirac segment. The dashed line in (a) shows that this pattern may belong to a polygon segment containing an angle $\theta$ greater than $\frac{\pi}{4}+\arctan 2 \approx 108^{\circ}$. Thus, our method may fail to detect a polygon angle greater than $108^{\circ}$.

When $x=0$, we have

$$
\mathcal{W}_{s} \theta(0)=C_{1} \phi+C_{2} s\left(k_{1}-k_{2}\right)
$$

where $C_{1}$ and $C_{2}$ are constants. Thus, the modulus maxima line occurs as a straight line of $s$ at $x=0$, and the modulus on the 


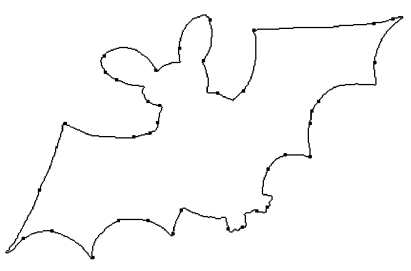

(a)

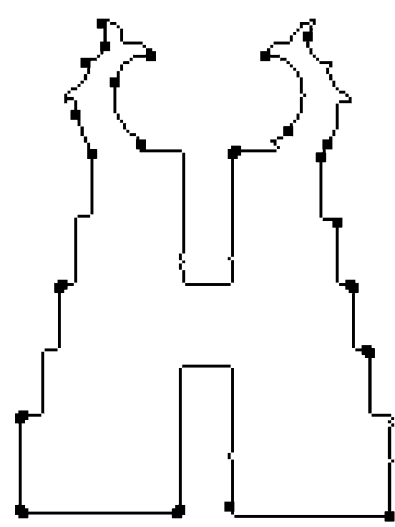

(c)

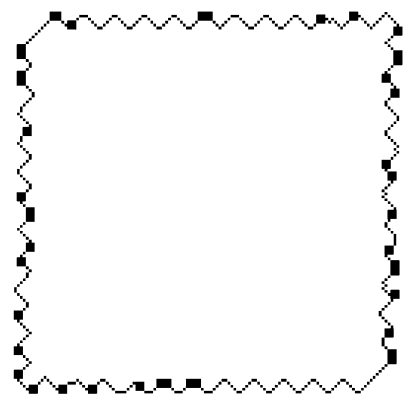

(e)

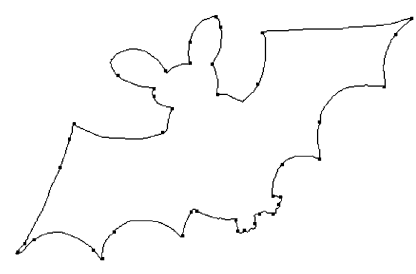

(b)

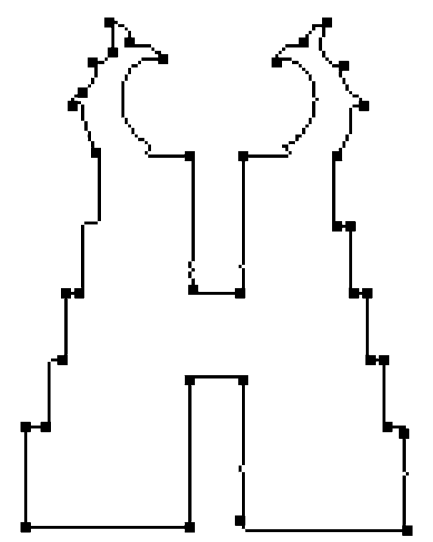

(d)

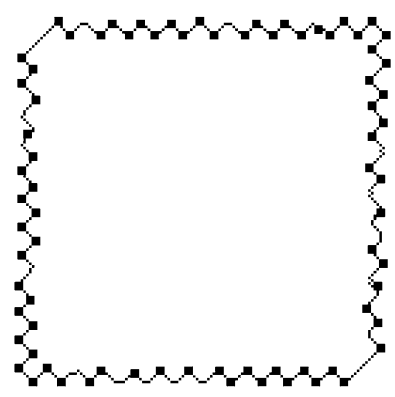

(f)
Fig. 6. The corners (marked by dots) obtained using wavelet modulus maxima. Left: from the real wavelet. Right: from the complex wavelet. Top: Bat. Middle: Temple. Bottom: Handkerchief.

In this model, the modulus value of the corner will not decrease as the scale $s$ increases. This implies that the Lipschitz regularity of the corner is a nonnegative value.

Equality (9) characterizes the Lipschitz regularity of a corner of a planar object with wavelet modulus maxima. In Appendix V, we show that the chain code of an imaged line is a constant, plus a Dirac impulse sequence

$$
\theta_{0}+\sum_{i} d_{i} \delta\left[n_{i}\right]
$$

where $\theta_{0}$ is an integer constant between 0 and $7, d_{i} \in\{-1,1\}$, and $\left|n_{i}-n_{i+1}\right| \geq 2$. Note that the chain code of a line is a constant value; thus, the above Dirac sequence results from the digitization of the line. A Dirac impulse has a negative Lipschitz regularity whose value is -1 . Therefore, the Dirac sequence can be removed by a singularity method that applies a threshold on the Lipschitz exponent so that a singularity of the Lipschitz exponent greater than the threshold is sustained. Although applying a threshold on the Lipschitz exponent effectively removes a Dirac sequence, it may also remove some corner singularities of a planar object. Fig. 5 shows some patterns whose corresponding chain codes are a five-point Dirac segment $(a, a, a \pm d, a, a)$ with $d \in\{1,2,3\}$. Any corner in a curved segment that yields one of these patterns after digitization cannot be detected by our method because the singularity of the corner is a Dirac and will be removed by our method.

In Fig. 6, we show and compare corner detection results (indicated by dots). Fig. 6 (a), (c) (e) shows the results obtained by superimposing the corners detected from applying real wavelet transform modulus analysis, while Fig. 6 (b), (d), (f) superimposes detected corners by applying complex-valued wavelet transform modulus analysis. The top pattern is a bat, the middle is a temple, and the bottom is a handkerchief. In our implementation, the Lipschitz exponent $\alpha$ of a maxima line was computed using the least median square regression method with the scale $s$ ranging from $2^{2}$ to $2^{6}$. We kept maxima lines having $\alpha \geq-0.5$ and deleted the rest. It is obvious that the corners of these patterns were better detected using the modulus maxima of a complex-valued wavelet.

\section{CONClusion}

We have proven that the wavelet transform modulus maxima of a complex-valued wavelet can be used to detect and characterize singularities. This is an extension of the previous work of Mallat and Hwang, which applied the modulus maxima using a real wavelet to detect singularities. Currently, analytic wavelets are used to measure instantaneous frequencies, while real wavelets are used for singularity detection. With our extension, we are able to simultaneously detect instantaneous frequencies and singularities using only the modulus maxima of the wavelet transform of an analytic wavelet. We have demonstrated the robustness of estimating singularities from the modulus maxima of an analytic wavelet over that of a real wavelet. Furthermore, we show that the singularity detection method can be employed to detect the corners of a planar object.

\section{APPENDIX I}

In this appendix, we prove Theorem 1 . We first need the following lemmas.

Lemma 1: Let $f(x)$ be a real-valued function and $\left|f^{\prime}(x)\right|>$ $C>0$ for $\forall x \in\left[t_{0}, t_{1}\right]$ then

$$
\int_{t_{0}}^{t_{1}}|f(x)| d x>\frac{1}{4}\left(t_{1}-t_{0}\right)^{2} C \text {. }
$$

Proof: Since $\left|f^{\prime}(x)\right|>C>0$, we know that $f^{\prime}(x)$ is a function with a constant sign in $\left[t_{0}, t_{1}\right]$. For simplicity, we only discuss the case where $f^{\prime}(x)$ are all positive in $\left[t_{0}, t_{1}\right]$. Since $f^{\prime}(x)$ are all positive, $f(x)$ is a strictly monotonically increasing function in $\left[t_{0}, t_{1}\right]$. We present our result by discussing the following two cases.

Case 1: $f(x)$ intersects with the $x$ axis.

Suppose $f(t)=0$ for $t \in\left[t_{0}, t_{1}\right]$. Since $\int_{t}^{x} f^{\prime}(x) d x=f(x)$, we have $f(x)>C(x-t)$. Therefore,

$$
\int_{t}^{t_{1}} f(x) d x>\frac{1}{2} C\left(t_{1}-t\right)^{2} \text {. }
$$

By similar deduction, we have $-\int_{t_{0}}^{t} f(x) d x>\frac{1}{2} C\left(t-t_{0}\right)^{2}$. Then

$$
\int_{t_{0}}^{t_{1}}|f(x)| d x>\frac{1}{2}\left(\left(t-t_{0}\right)^{2} C+\left(t_{1}-t\right)^{2} C\right) \geq \frac{1}{4} C\left(t_{1}-t_{0}\right)^{2} .
$$


Case 2: $f(x)$ does not intersect with the $x$ axis.

Suppose $f(x)>0$ for all $x \in\left[t_{0}, t_{1}\right]$. Since

$$
f(x)-f\left(t_{0}\right)=\int_{t_{0}}^{x} f^{\prime}(x) d x>C\left(x-t_{0}\right)
$$

we have

$$
\int_{t_{0}}^{t_{1}} f(x) d x>\int_{t_{0}}^{t_{1}} f(x) d x-f\left(t_{0}\right)\left(t_{1}-t_{0}\right)>\frac{1}{2} C\left(t_{1}-t_{0}\right)^{2} .
$$

Similarly, let $f(x)<0$ for $x \in\left[t_{0}, t_{1}\right]$. Since

$$
f\left(t_{1}\right)-f(x)=\int_{x}^{t_{1}} f^{\prime}(x) d x<C\left(t_{1}-x\right)
$$

we have

$-\int_{t_{0}}^{t_{1}} f(x) d x>-\int_{t_{0}}^{t_{1}} f(x) d x+f\left(t_{1}\right)\left(t_{1}-t_{0}\right)>\frac{1}{2} C\left(t_{1}-t_{0}\right)^{2}$.

Based on Cases 1 and 2, we have

$$
\int_{t_{0}}^{t_{1}}|f(x)| d x>\frac{1}{4}\left(t_{1}-t_{0}\right)^{2} C .
$$

Lemma 2: Let $f(x)$ be a real-valued function. If

$$
\forall x \in\left[t_{0}, t_{1}\right], \quad\left|f^{\prime \prime}(x)\right|>C>0
$$

then

$$
\int_{t_{0}}^{t_{1}}|f(x)| d x>\frac{1}{16}\left(\frac{t_{1}-t_{0}}{3}\right)^{3} C .
$$

Proof: Since $f^{\prime \prime}(x)$ is a constant sign function in $\left[t_{0}, t_{1}\right]$, $f^{\prime}(x)$ is a strictly monotonically increasing or decreasing function in $\left[t_{0}, t_{1}\right]$. We then have

$$
\begin{aligned}
&\left(\frac{t_{1}-t_{0}}{3}\right) \max \left(\left|f^{\prime}\left(t_{0}+\frac{1}{3}\left(t_{1}-t_{0}\right)\right)\right|,\right.\left.\left|f^{\prime}\left(t_{0}+\frac{2}{3}\left(t_{1}-t_{0}\right)\right)\right|\right) \\
& \geq \int_{t_{0}+\frac{1}{3}\left(t_{1}-t_{0}\right)}^{t_{0}+\frac{2}{3}\left(t_{1}-t_{0}\right)}\left|f^{\prime}(x)\right| d x>\frac{1}{4}\left(\frac{t_{1}-t_{0}}{3}\right)^{2} C .
\end{aligned}
$$

The last inequality above follows from Lemma 1 . Without loss of generality, let us suppose that

$$
\begin{aligned}
\max \left(\left|f^{\prime}\left(t_{0}+\frac{1}{3}\left(t_{1}-t_{0}\right)\right)\right|, \mid f^{\prime}\right. & \left.\left(t_{0}+\frac{2}{3}\left(t_{1}-t_{0}\right)\right) \mid\right) \\
& =\left|f^{\prime}\left(t_{0}+\frac{2}{3}\left(t_{1}-t_{0}\right)\right)\right|
\end{aligned}
$$

Then, $f^{\prime}(x)$ must be either all positive values or all negative values within the interval $\left[t_{0}+\frac{2}{3}\left(t_{1}-t_{0}\right), t_{1}\right]$. If this is not the case, then

$$
\left|f^{\prime}\left(t_{0}+\frac{1}{3}\left(t_{1}-t_{0}\right)\right)\right|>\left|f^{\prime}\left(t_{0}+\frac{2}{3}\left(t_{1}-t_{0}\right)\right)\right|
$$

and a violation of our assumption occurs. Thus, we have for any $x \in\left[t_{0}+\frac{2}{3}\left(t_{1}-t_{0}\right), t_{1}\right]$

$$
\left|f^{\prime}(x)\right|>\left|f^{\prime}\left(t_{0}+\frac{2}{3}\left(t_{1}-t_{0}\right)\right)\right|>0 .
$$

By Lemma 1,

$$
\begin{aligned}
& \int_{t_{0}+\frac{2}{3}\left(t_{1}-t_{0}\right)}^{t_{1}}|f(x)| d x \\
& \quad>\frac{1}{4}\left(\frac{t_{1}-t_{0}}{3}\right)^{2}\left|f^{\prime}\left(t_{0}+\frac{2}{3}\left(t_{1}-t_{0}\right)\right)\right| \\
& \quad \geq \frac{1}{4}\left(\frac{t_{1}-t_{0}}{3}\right)^{3} \frac{1}{4} C=\frac{1}{16}\left(\frac{t_{1}-t_{0}}{3}\right)^{3} C .
\end{aligned}
$$

Therefore,

$$
\int_{t_{0}}^{t_{1}}|f(x)| d x>\int_{t_{0}+\frac{2}{3}\left(t_{1}-t_{0}\right)}^{t_{1}}|f(x)| d x>\frac{1}{16}\left(\frac{t_{1}-t_{0}}{3}\right)^{3} C .
$$

Lemma 3: Let $K$ be a positive constant, and let $g(x)$ be a complex function that satisfies $\int_{c}^{d}|g(x)| d x<K$. Suppose that $\left|\frac{d g(x)}{d x}\right|$ has no local maxima in $[c, d]$. Let $\beta>0$, and let the first derivatives of $\operatorname{Re}(g(x))$ and $\operatorname{Im}(g(x))$ be finite deviations; then there exists a constant $C_{\beta}$ such that, for all $x$ in $[c+\beta, d-\beta]$, $\left|\frac{d g(x)}{d x}\right|<C_{\beta}$.

Proof: Since $\left|g^{\prime}(x)\right|$ has no local maxima in $[c, d]$, for all $x$ in $[c+\beta, d-\beta]$

$$
\left|g^{\prime}(x)\right|<\max \left(\left|g^{\prime}(c+\beta)\right|,\left|g^{\prime}(d-\beta)\right|\right) .
$$

Without loss of generality, let us suppose that

$$
\max \left(\left|g^{\prime}(c+\beta)\right|,\left|g^{\prime}(d-\beta)\right|\right)=g^{\prime}(c+\beta) .
$$

Let $g(x)=a(x)+i b(x)$, where $a(x)=\operatorname{Re}(g(x))$ and $b(x)=\operatorname{Im}(g(x))$ are real functions. Since the first derivatives of $\operatorname{Re}(g(x))$ and $\operatorname{Im}(g(x))$ are finite deviations, we can divide the interval $\left[c+\frac{\beta}{2}, c+\beta\right]$ into $M(\beta)$ subintervals $v_{1}, v_{2}, \ldots, v_{M}$. Within each interval $v_{i}$ : either $a^{\prime}(x)=b^{\prime}(x)$ for all $x$ in $v_{i}$, or $a^{\prime}(x)=b^{\prime}(x)$ only at the two ends of the subinterval $v_{i}$. We now define a real function $c(x)$ over $\left[c+\frac{\beta}{2}, c+\beta\right]$ such that

$$
c(x)= \begin{cases}a(x), & \text { if }\left|a^{\prime}(x)\right| \geq\left|b^{\prime}(x)\right| \\ b(x), & \text { if }\left|b^{\prime}(x)\right|>\left|a^{\prime}(x)\right| .\end{cases}
$$

As a result, we have

$$
\left|c^{\prime}(x)\right| \geq \frac{\left|g^{\prime}(x)\right|}{\sqrt{2}}>\frac{\left|g^{\prime}(c+\beta)\right|}{\sqrt{2}}>0
$$

for $x \in\left[c+\frac{\beta}{2}, c+\beta\right]$. We can now apply Lemma 1 to each interval $v_{i}, i=1,2, \ldots, M(\beta)$ over $\left[c+\frac{\beta}{2}, c+\beta\right]$ and obtain

$$
\int_{v_{i}}|c(x)| d x>\frac{1}{4}\left|v_{i}\right|^{2} \frac{\left|g^{\prime}(c+\beta)\right|}{\sqrt{2}}
$$


where $\left|v_{i}\right|$ is the length of the subinterval $v_{i}$. Furthermore, we have

$$
\begin{aligned}
K & >\int_{c}^{d}|g(x)| d x>\int_{c+\frac{\beta}{2}}^{c+\beta}|c(x)| d x=\sum_{i} \int_{v_{i}}|c(x)| d x \\
& >\sum_{i}\left|v_{i}\right|^{2} \frac{\left|g^{\prime}(c+\beta)\right|}{4 \sqrt{2}} .
\end{aligned}
$$

Clearly

$$
\left(\sum_{i}\left|v_{i}\right|^{2}\right) M(\beta) \geq\left(\sum_{i}\left|v_{i}\right|\right)^{2}=\frac{\beta^{2}}{4} .
$$

Therefore,

$$
\sum_{i}\left|v_{i}\right|^{2} \geq \frac{\beta^{2}}{4 M(\beta)}
$$

Substituting this into (11), we have

$$
K>\frac{1}{4 \sqrt{2}} \frac{\beta^{2}}{4 M(\beta)}\left|g^{\prime}(c+\beta)\right|
$$

and, therefore,

$$
\left|g^{\prime}(c+\beta)\right|<\frac{16 \sqrt{2} M(\beta) K}{\beta^{2}}=C_{\beta} .
$$

As a result, for all $x$ in $[c+\beta, d-\beta],\left|g^{\prime}(x)\right|<C_{\beta}$.

Lemma 4: Let $K$ be a positive constant, and let $g(x)$ be a complex-valued function that satisfies

$$
\int_{c}^{d}|g(x)| d x<K
$$

Suppose that $\left|\frac{d^{2} g(x)}{d x^{2}}\right|$ has no local maxima in $[c, d]$. Let $\beta>$ 0 , and let the second derivatives of $\operatorname{Re}(g(x))$ and $\operatorname{Im}(g(x))$ be finite deviations. Then, a constant $D_{\beta}$ exists such that for all $x$ in $[c+\beta, d-\beta],\left|\frac{d^{2} g(x)}{d x^{2}}\right|<D_{\beta}$.

Proof: The proof of this lemma is similar to that of the previous lemma. $\left|g^{\prime \prime}(x)\right|$ has no local maxima for all $x$ in $[c, d]$. Therefore, for all $x$ in $(c+\beta, d-\beta)$

$$
\left|g^{\prime \prime}(x)\right| \leq \max \left(\left|g^{\prime \prime}(c+\beta)\right|,\left|g^{\prime \prime}(d-\beta)\right|\right) .
$$

Without loss of generality, let us suppose that $\max \left(\mid g^{\prime \prime}(c+\right.$ $\left.\beta)|,| g^{\prime \prime}(d-\beta) \mid\right)$ is equal to $\left|g^{\prime \prime}(c+\beta)\right|$.

Let $g(x)=a(x)+i b(x)$, where $a(x)=\operatorname{Re}(g(x))$ and $b(x)=\operatorname{Im}(g(x))$ are real functions. Since the second derivatives of $\operatorname{Re}(g(x))$ and $\operatorname{Im}(g(x))$ are finite deviations, we can divide $\left[c+\frac{\beta}{2}, c+\beta\right]$ into $M(\beta)$ subintervals $v_{1}, v_{2}, \ldots, v_{M}$. Within each subinterval $v_{i}$ : either $a^{\prime \prime}(x)=b^{\prime \prime}(x)$ for all $x$ in the subinterval, or $a^{\prime \prime}(x)=b^{\prime \prime}(x)$ only at the two ends of the subinterval. Let a real function $c(x)$ be

$$
c(x)= \begin{cases}a(x), & \text { if }\left|a^{\prime \prime}(x)\right| \geq\left|b^{\prime \prime}(x)\right| \\ b(x), & \text { if }\left|b^{\prime \prime}(x)\right|>\left|a^{\prime \prime}(x)\right| .\end{cases}
$$

Then, we have

$$
\left|c^{\prime \prime}(x)\right| \geq \frac{\left|g^{\prime \prime}(x)\right|}{\sqrt{2}}>\frac{\left|g^{\prime \prime}(c+\beta)\right|}{\sqrt{2}}>0
$$

for $x \in\left[c+\frac{\beta}{2}, c+\beta\right]$. For a subinterval $v_{i}$, by Lemma 2, we have

$$
\int_{v_{i}}|c(x)| d x>\frac{1}{16}\left(\frac{\left|v_{i}\right|}{3}\right)^{3} \frac{\left|g^{\prime \prime}(c+\beta)\right|}{\sqrt{2}} .
$$

Furthermore, we have

$$
\begin{aligned}
K & >\int_{c}^{d}|g(x)| d x>\int_{c+\frac{\beta}{2}}^{c+\beta}|c(x)| d x=\sum_{i} \int_{v_{i}}|c(x)| d x \\
& >\sum_{i} \frac{1}{16}\left(\frac{\left|v_{i}\right|}{3}\right)^{3} \frac{\left|g^{\prime \prime}(c+\beta)\right|}{\sqrt{2}} .
\end{aligned}
$$

Assume that $\beta<1$. Then, all $\left|v_{i}\right|<1$ and, therefore,

$$
\sum_{i}\left|v_{i}\right|^{3} \geq \sum_{i}\left|v_{i}\right|^{4}
$$

Clearly,

$$
\sum_{i}\left|v_{i}\right|^{4} \geq \frac{\left(\sum_{i}\left|v_{i}\right|^{2}\right)^{2}}{M(\beta)}
$$

Then, we have

$$
\sum_{i}\left|v_{i}\right|^{3} \geq \sum_{i}\left|v_{i}\right|^{4} \geq \frac{\left(\sum_{i}\left|v_{i}\right|^{2}\right)^{2}}{M(\beta)} \geq \frac{\beta^{4}}{16(M(\beta))^{2}} .
$$

Substituting this into (12), we have

$$
K>\frac{1}{16} \frac{1}{3^{3}} \frac{\beta^{4}}{16(M(\beta))^{2}} \frac{\left|g^{\prime \prime}(c+\beta)\right|}{\sqrt{2}} .
$$

Then, we have $\left|g^{\prime \prime}(c+\beta)\right|<\frac{2^{8} 3^{3} \sqrt{2}(M(\beta))^{2} K}{\beta^{2}}=D_{\beta}$. Thus, we have for all $x$ in $[c+\beta, d-\beta],\left|g^{\prime \prime}(x)\right|<D_{\beta}$.

\section{Proof of Theorem 1}

1. We prove the first part of this theorem by induction with the following proposition.

Proposition $\left(P_{n}\right)$ : Let $\Psi(x)$ be a complex-valued wavelet that can be written as $\Psi(x)=d^{n} \Phi(x) / d x^{n}$. $\Phi(x)$ is equal to $\Phi_{R}(x)+i \Phi_{I}(x)$, where both $\Phi_{R}(x)$ and $\Phi_{I}(x)$ are continuous functions of compact support. Let $f(x)$ be a real function. Then, for any $\epsilon>0$, a constant $K_{\epsilon}$ exists such that, at all scales $s$

$$
\int_{a+\epsilon}^{b-\epsilon}\left|f * \Phi_{s}(x)\right| d x \leq K_{\epsilon}
$$

where $\Phi_{s}(x)=\frac{1}{s} \Phi\left(\frac{x}{s}\right)$.

If $\mathcal{W} f(s, x)$ has no modulus maxima for $x \in(a, b)$ and $s<$ $s_{0}$, then for any $\epsilon>0$, there exists a constant $A_{\epsilon, n}$ such that, for any $x \in(a+\epsilon, b-\epsilon)$ and $s<s_{0}$

$$
|\mathcal{W} f(s, x)| \leq A_{\epsilon, n} s^{n} .
$$

Proof: If we modify $f(x)$ by multiplying it by the indicator function of $[a, b]$, then we do not modify its regularity on 
any interval $[a+\epsilon, b-\epsilon]$. In the following, we thus assume that $f(x)=0$ for all $x \notin[a, b]$.

- Let us first prove that (13) is satisfied.

Since $f(x) \in L^{1}([a, b])$ and $f(x)=0$ for all $x \notin[a, b]$, it follows that

$$
\int_{a}^{b}\left|f * \Phi_{s}(x)\right| d x \leq \int_{a}^{b}|f(x)| d x \int_{-\infty}^{+\infty}\left|\Phi_{s}(x)\right| d x .
$$

With a change of variable in the integral, we obtain

$$
\begin{aligned}
\int_{-\infty}^{+\infty}\left|\Phi_{s}(x)\right| d x & =\int_{-\infty}^{+\infty}|\Phi(x)| d x \\
& \leq \int_{-\infty}^{+\infty}\left|\Phi_{R}(x)\right| d x+\int_{-\infty}^{+\infty}\left|\Phi_{I}(x)\right| d x .
\end{aligned}
$$

Hence, $\int_{a}^{b}\left|f * \Phi_{s}(x)\right| d x$ is bounded by a constant independent of the scale $s$, as in (13).

- Let us now prove that (14) is satisfied.

We first prove that the proposition is true for $n=1$. Since $\Psi(x)=d \Phi(x) / d x$, we find that

$$
\mathcal{W} f(s, x)=s \frac{d}{d x}\left(f * \Phi_{s}\right)(x) .
$$

Let $g(x)=\left(f * \Phi_{s}\right)(x)$. By (13) and Lemma 3, we have for all $x \in[c+\epsilon, d-\epsilon],\left|\frac{d}{d x}\left(f * \Phi_{s}\right)(x)\right|<A_{\epsilon, 1}$. Therefore, $|\mathcal{W} f(s, x)| \leq s A_{\epsilon, 1}$.

The proof of $\left(P_{n}\right)$ for $n=2$ is based on (13) and Lemma 4. Since $\Psi(x)=d^{2} \Phi(x) / d x^{2}$, we find that

$$
\mathcal{W} f(s, x)=s^{2} \frac{d^{2}}{d x^{2}}\left(f * \Phi_{s}\right)(x) .
$$

For all $x \in[c+\epsilon, d-\epsilon],\left|\frac{d^{2}}{d x^{2}}\left(f * \Phi_{s}\right)(x)\right|<A_{\epsilon, 2}$. Therefore, $|\mathcal{W} f(s, x)| \leq s^{2} A_{\epsilon, 2}$.

- Let us now prove that if $\left(P_{n}\right)$ is true for $n \geq 2$, then $\left(P_{n+1}\right)$ is also true. Let $\Psi(x)$ be a wavelet with $n+1$ vanishing moments. The wavelet $\Psi(x)$ can be written $\Psi(x)=d \chi(x) / d x$, where $\chi(x)$ is a wavelet that has $n$ vanishing moments. Let $d f(x) / d x$ be the derivative of $f(x)$ in terms of distributions; then

$$
\mathcal{W} f(s, x)=s \frac{d f}{d x} * \chi_{s}(x) .
$$

In order to apply our induction hypothesis $\left(P_{n}\right)$ to $d f(x) / d x$ with respect to the wavelet $\chi(x)$, we need to prove that for any $\epsilon>0$, a constant $K_{\epsilon}$ exists such that, at all scales $s$

$$
\int_{a+\epsilon}^{b-\epsilon}\left|\frac{d f}{d x} * \Phi_{s}(x)\right| d x \leq K_{\epsilon} .
$$

Since the wavelet $\Psi(x)$ has more than two vanishing moments, proposition $\left(P_{2}\right)$, which we proved above, implies that for any $\epsilon>0$ if $x \in(a+\epsilon, b-\epsilon)$, then

$$
|\mathcal{W} f(s, x)| \leq s^{2} A_{\epsilon, 2} .
$$

Assuming that $\Psi(x)=\Psi_{R}(x)+i \Psi_{I}(x)$, and that $\Psi_{R}(x)$, $\Psi_{I}(x), d \Psi_{R}(x) / d x$, and $d \Psi_{I}(x) / d x$ are all in $L^{1}(R)$, then $f(x)$ is uniformly Lipschitz $\alpha$ on the intervals $(a+$ $\epsilon, b-\epsilon$ ), for any $\alpha<2$ [10], [17], [18] (also Appendix III).
Hence, $d f(x) / d x$ is uniformly bounded on any such interval. We can then easily derive that (16) is satisfied. Let us now apply the induction hypothesis $\left(P_{n}\right)$ to $d f(x) / d x$ with respect to the wavelet $\chi(x)$. A constant $A_{\epsilon, n}$ exists such that for any $x \in(a+\epsilon, b-\epsilon)$ and $s<s_{0}$

$$
\left|\frac{d f}{d x} * \chi_{s}(x)\right| \leq A_{\epsilon, n} s^{n} .
$$

Equation (15) implies that

$$
|\mathcal{W} f(s, x)| \leq A_{\epsilon, n} s^{n+1} .
$$

This completes the proof of $\left(P_{n+1}\right)$.

By applying the theorem stated in Appendix III to statement $\left(P_{n}\right)$, we derive that the function $f(x)$ is Lipschitz $\alpha$ for any $\alpha<n$.

2. The proof of the second part of Theorem 1 is as follows.

- Let us now prove that (14) implies that $f(x)$ is Lipschitz $n$ if the wavelet $\Psi(x)$ can be written as

$$
\Psi(x)=\frac{d^{n} \theta(x)}{d x}
$$

where $\theta(x)=\theta_{R}(x)+i \theta_{I}(x)$, and both $\theta_{R}(x)$ and $\theta_{I}(x)$ are smoothing functions. Let $d^{n} f(x) / d x^{n}$ be the $n$th derivative of $f(x)$ in terms of distributions. Similar to (15), (17) yields

$$
\mathcal{W} f(s, x)=s^{n} \frac{d^{n} f}{d x^{n}} * \theta_{s}(x) .
$$

Equation (14) of proposition $\left(P_{n}\right)$ implies that for any $\epsilon>0$, there exists a constant $A_{\epsilon, n}$ such that for any $x \in$ $(a+\epsilon, b-\epsilon)$ and $s<s_{0}$

$$
\begin{aligned}
\left|\frac{d^{n} f}{d x^{n}} * \theta_{s}(x)\right| & \left.\left.\leq \mid \frac{d^{n} f}{d x^{n}} *\left(\theta_{R}\right)_{s}(x)\right)|+| \frac{d^{n} f}{d x^{n}} *\left(\theta_{I}\right)_{s}(x)\right) \mid \\
& \leq\left(A_{R}\right)_{\epsilon, n}+\left(A_{I}\right)_{\epsilon, n} \\
& \leq A_{\epsilon, n} .
\end{aligned}
$$

Since the integral of $\theta(x)$ is nonzero, this equation implies that $d^{n} f(x) / d x^{n}$ is a function that is bounded by $A_{\epsilon, n}$ over the interval $(a+\epsilon, b-\epsilon)$. Hence, $f(x)$ is uniformly Lipschitz $n$ over the interval $(a+\epsilon, b-\epsilon)$.

\section{APPENDIX II \\ PROOF OF THEOREM 2}

First, we derive that $f(x)$ is Lipschitz $n$ at any $x \neq x_{0}$. For any $\epsilon>0$, a scale $s_{\epsilon}$ exists such that for all $s<s_{\epsilon}$, there is no wavelet modulus maxima at $\left(a+\epsilon, x_{0}-\epsilon\right)$. Furthermore, from Theorem 1, the $n$th derivatives of $\operatorname{Re}(\mathcal{W} f(s, x))$ and $\operatorname{Im}(\mathcal{W} f(s, x))$ are finite deviations for all $s$; therefore, the Lipschitz regularity in the neighborhood of any $x_{1} \in\left(a+\epsilon, x_{0}-\epsilon\right)$ is $n$. Thus, $f(x)$ is uniform Lipschitz $n$ at any point in $\left(a, x_{0}\right)$. The same is true for any point in $\left(x_{0}, b\right)$.

Let us prove that $f(x)$ is Lipschitz $\alpha$ at $x_{0}$, and that it can be characterized by the complex-valued wavelet modulus maxima. For any interval $\left(x_{0}-\epsilon, x_{0}+\epsilon\right)$ including $x_{0}$, there is a small 
enough scale $s_{\epsilon}$ such that for all $s<s_{\epsilon}$ the wavelet modulus maxima for these scales are contained entirely within the region $\left(x_{0}-\epsilon, x_{0}+\epsilon\right)$ by $\left(0, s_{\epsilon}\right)$. Then, there is a constant $D_{\epsilon}$ such that all the wavelet transforms in the region will be bounded by

$$
|\mathcal{W} f(s, x)|<D_{\epsilon} s^{\alpha}
$$

From the theorem stated in Appendix III, any subinterval including $x_{0}$ in $\left(x_{0}-\epsilon, x_{0}+\epsilon\right)$ will be Lipschitz $\alpha$. This implies that the Lipschitz regularity at $x_{0}$ is $\alpha$.

\section{APPENDIX III}

Here, we review a well-known theorem, a proof of which can be found in [10], [17], [18].

Theorem: Let $f(x)$ be a real-valued function, and let $[a, b]$ be an interval of $R$. Let $0<\alpha<1$. Then, $f(x)$ is uniformly Lipschitz $\alpha$ over $(a+\epsilon, b-\epsilon)$ if and only if for any $\epsilon>0$, a constant $A_{\epsilon}$ exists such that for all $x \in(a+\epsilon, b-\epsilon)$ and $s>0$, $|\mathcal{W} f(s, x)| \leq A_{\epsilon} s^{\alpha}$.

One can easily show that the theorem is also true for a complex-valued wavelet $\Psi(x)=\Psi_{R}(x)+i \Psi_{I}(x)$, provided that $\Psi_{R}(x), \Psi_{I}(x), d \Psi_{R}(x) / d x$, and $d \Psi_{I}(x) / d x$ are all in $L^{1}(R)$. This theorem can also be extended to a Lipschitz exponent $\alpha$ larger than 1 by requiring the wavelet to have enough vanishing moments. A related discussion can be found in [14].

\section{APPENDIX IV}

In this appendix, we analyze the signal

$$
f(t)=-|t|^{\beta}(u(t+1)-u(t))-|t|^{\alpha}(u(t)-u(t-1))
$$

using a second derivative Gaussian wavelet $\psi^{r}(t)$ and an analytic wavelet whose real part is $\psi^{r}(t)$.

We first show that there are three maxima lines if the wavelet is $\psi^{r}(t)$. Let the wavelet transform in scale $s$ be

$$
f * \psi_{s}^{r}(t)=s^{2} f * \frac{d^{2} \theta_{s}(t)}{d t^{2}} .
$$

Clearly, $f * \psi_{s}^{r}(t) \geq 0$ for all $t$. Thus, the maxima lines can be obtained by taking the derivative of $f * \psi_{s}^{r}(t)$ and setting the result to zero. Omitting the constant, we have

$$
\begin{aligned}
\frac{d\left(f * \psi_{s}^{r}(t)\right)}{d t}= & \int_{0}^{1} \alpha(\alpha-1) a^{\alpha-2} \frac{-(t-a)}{(s \sigma)^{3}} e^{-\frac{(t-a)^{2}}{2(s \sigma)^{2}}} d a \\
& +\int_{-1}^{0} \beta(\beta-1)(-a)^{\beta-2} \frac{-(t-a)}{(s \sigma)^{3}} e^{-\frac{(t-a)^{2}}{2(s \sigma)^{2}}} d a \\
= & 0
\end{aligned}
$$

where $\sigma$ is the standard deviation of the Gaussian function. For each $t$, if the scale is large enough such that $\frac{t}{s \sigma} \approx 0$, then

$$
e^{-\frac{(t-a)^{2}}{2(s \sigma)^{2}}} \approx 1-\frac{(t-a)^{2}}{2(s \sigma)^{2}}
$$

Substituting this into (18) and calculating the integrations, we obtain a degree 3 polynomial of $\frac{t}{s \sigma}$

$$
\begin{aligned}
p\left(\frac{t}{s \sigma}\right)=c_{3}(\alpha, \beta)\left(\frac{t}{s \sigma}\right)^{3} & +c_{2}(\alpha, \beta)\left(\frac{t}{s \sigma}\right)^{2} \\
& +c_{1}(\alpha, \beta)\left(\frac{t}{s \sigma}\right)+c_{0}(\alpha, \beta)=0 .
\end{aligned}
$$

By substituting $\alpha=0.6$ and $\beta=0.2$, which correspond to the leftmost singularity in Fig. 1, we derive three real roots. Note that when $\alpha=\beta$, the terms $c_{0}$ and $c_{2}$ in $p\left(\frac{t}{s \sigma}\right)$ are zeros. This indicates that $t=0$ is a real root; and if $\frac{t_{0}}{s_{0} \sigma}$ is a root, then $-\frac{t_{0}}{s_{0} \sigma}$ is also a root for such a case.

Now, we will show that there is only one maxima line when we use the analytical wavelet $\psi(t)=\psi^{r}(t)+j \psi^{i}(t)$, whose real part is the second derivative of the Gaussian function. The Hilbert transform of $\psi^{r}(t)=\left(t^{2}-1\right) e^{-\frac{t^{2}}{2}}$ is

$$
\begin{aligned}
\psi^{i}(t)= & \frac{1}{\pi} \int_{-\infty}^{\infty} \frac{a^{2}-1}{a-t} e^{-\frac{a^{2}}{2}} d a \\
= & \frac{1}{\pi}\left(t \int_{-\infty}^{\infty} e^{-\frac{a^{2}}{2}} d a+\int_{-\infty}^{\infty} a e^{-\frac{a^{2}}{2}} d a\right. \\
& \left.\quad+\left(t^{2}-1\right) \int_{-\infty}^{\infty} \frac{e^{-\frac{a^{2}}{2}}}{a-t} d a\right) \\
= & t \sqrt{\frac{2}{\pi}}+\left(t^{2}-1\right) \frac{1}{\pi} \int_{-\infty}^{\infty} \frac{e^{-\frac{a^{2}}{2}}}{a-t} d a .
\end{aligned}
$$

The Hilbert transform of a Gaussian function is a confluent hyper-geometric function, which has a hyper-geometric series and generally does not have a closed form. However, we can approximate the Hilbert transform of a Gaussian function $e^{-\frac{1}{2}\left(\frac{t}{s \sigma}\right)^{2}}$ when $\frac{t}{s \sigma} \approx 0$

$$
e^{-\frac{1}{2}\left(\frac{t}{s \sigma}\right)^{2}} \approx 1-\frac{\left(\frac{t}{s \sigma}\right)^{2}}{2} \approx \cos \left(\frac{t}{s \sigma}\right) .
$$

The Hilbert transform of $\cos \left(\frac{t}{s \sigma}\right)$ is $-\sin \left(\frac{t}{s \sigma}\right)$, which is also the derivative of $\cos \left(\frac{t}{s \sigma}\right)$ with respect to $\frac{t}{s \sigma}$. Therefore, when $\frac{t}{s \sigma} \approx 0$, the Hilbert transform of $e^{-\frac{1}{2}\left(\frac{t}{s \sigma}\right)^{2}}$ can be approximated as its derivative and yields $-\frac{t}{s \sigma} e^{-\frac{1}{2}\left(\frac{t}{s \sigma}\right)^{2}}$. Substituting this result into the last term in (19), we obtain

$$
\begin{aligned}
\psi^{i}(t) & =\frac{1}{\pi}\left(\sqrt{2 \pi} t+\left(t-t^{3}\right) e^{-\frac{t^{2}}{2}}\right) \\
& =\frac{1}{\pi}\left(\sqrt{2 \pi} t-\left(e^{-\frac{t^{2}}{2}}\right)^{\prime \prime \prime}+2\left(e^{-\frac{t^{2}}{2}}\right)^{\prime}\right) .
\end{aligned}
$$

The above is the imaginary part of our analytical wavelet $\psi(t)$. The wavelet transform of $f(t)$ is

$$
\begin{aligned}
f * \psi_{s}(t) & =s^{2} f * \theta_{s}^{\prime \prime}(t) \\
& +j \frac{-1}{\pi}\left(f * s^{3} \theta_{s}^{\prime \prime \prime}(t)+2 s \theta_{s}^{\prime}(t)-\sqrt{2 \pi} \frac{t}{s^{2}}\right) .
\end{aligned}
$$

Let $\operatorname{Re}(*)$ be the real part of $*$ and $\operatorname{Im}(*)$ be the imaginary part of $*$. The wavelet transform modulus maxima can be found by

$$
\begin{aligned}
\frac{d\left|f * \psi_{s}(t)\right|^{2}}{d t}=2\left[\operatorname{Re}\left(f * \psi_{s}(t)\right)\left(\operatorname{Re}\left(f * \psi_{s}(t)\right)\right)^{\prime}\right. \\
\left.\quad+\operatorname{Im}\left(f * \psi_{s}(t)\right)\left(\operatorname{Im}\left(f * \psi_{s}(t)\right)\right)^{\prime}\right] \\
=0 .
\end{aligned}
$$

Substituting (21) into the above equation, and supposing that $\frac{t}{s \sigma} \approx 0$ (this can be done for each $t$ by allowing scale $s$ to be large enough) and using the approximation

$$
e^{-\frac{(t-a)^{2}}{2(s \sigma)^{2}}} \approx 1-\frac{(t-a)^{2}}{2(s \sigma)^{2}}
$$




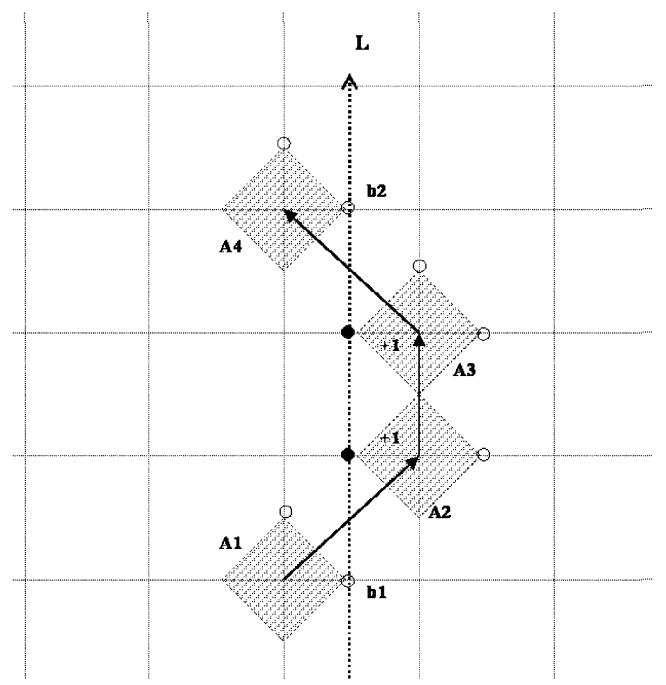

(a)

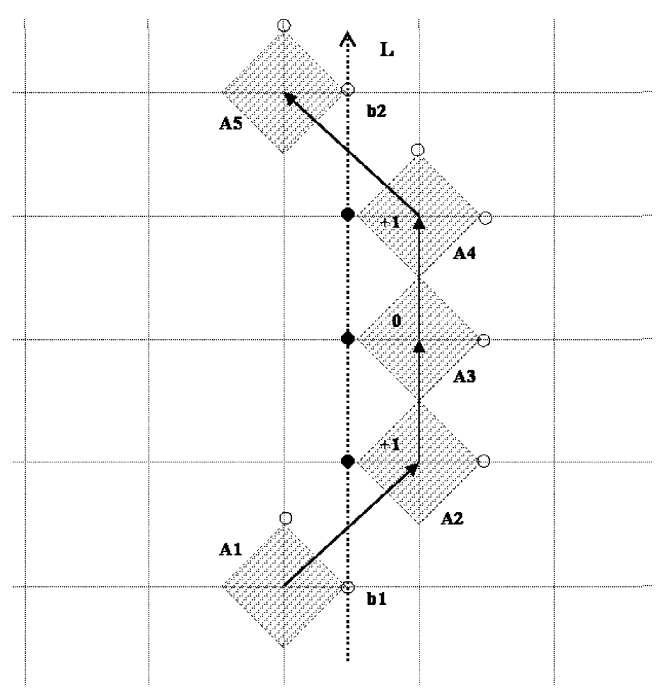

(b)

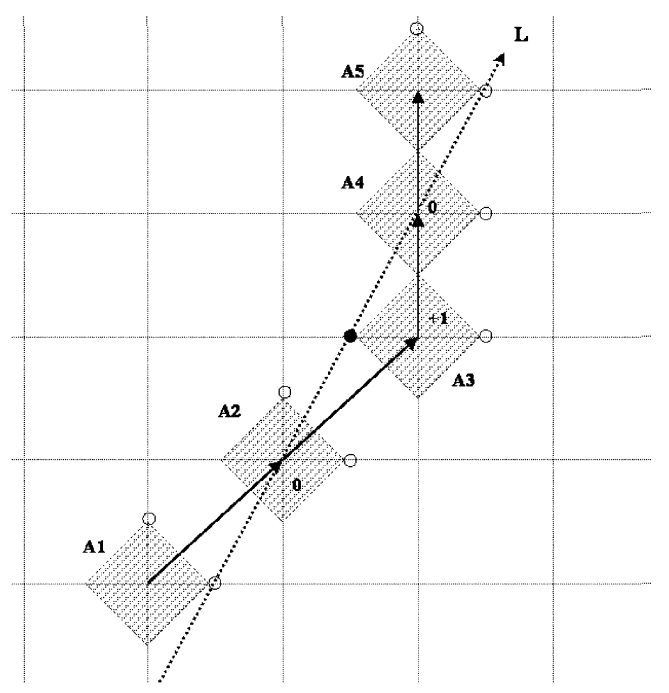

(c)

we obtain a polynomial of $\frac{t}{s \sigma}$ of degree 6 . Omitting any degree higher than 3 of this polynomial (because $\frac{t}{s \sigma} \approx 0$ ) yields the cubic equation

$$
\begin{aligned}
d_{3}(\alpha, \beta)\left(\frac{t}{s \sigma}\right)^{3}+d_{2}(\alpha, \beta)( & \left.\frac{t}{s \sigma}\right)^{2} \\
& +d_{1}(\alpha, \beta) \frac{t}{s \sigma}+d_{0}(\alpha, \beta)=0 .
\end{aligned}
$$

Using a similar technique to that used to obtain the roots of the real wavelet transform modulus maxima, we find numerically that the solution of the above cubic equation has a unique real root when $\alpha=0.6$ and $\beta=0.2$. When $\alpha=\beta, d_{0}$ is zero. In such a case, the real root is at $t=0$.

\section{APPENDIX V}

In this appendix, we show that the chain code of an imaged line is

$$
\theta_{0}+\sum_{i} d_{i} \delta\left[n_{i}\right]
$$

where $\theta_{0}$ is a constant, $d_{i} \in\{-1,1\}$, and $\left|n_{i}-n_{i+1}\right| \geq 2$.

Let $\theta[n]$ be the chain code of an imaged line, and its discrete derivative be $\Delta \theta[n]=\theta[n+1]-\theta[n]$. We show that the following are impossible patterns for $\Delta \theta[n]$. We use $(x)^{*}$ to denote a sequence of $x$ with length $\geq 0$. We shall show that $\Delta \theta[n]$ has the following two properties:

- P1: $\Delta \theta[n]$ contains neither a subsequence $\cdots 10^{*} 1 \cdots$ nor $\cdots(-1) 0^{*}(-1) \cdots$

Proof: The dashed diamond in Fig. 7 indicates that any point in the region will be quantized to the lattice point at the center of the diamond. We stipulate that the top and right coners of a diamond are not included in the dashed area.

Fig. 7 (a) shows that subsequence 11 is impossible in $\Delta \theta[n]$. The right corners of diamonds $A 1$ and $A 4$ are not quantized to our lattice point. Thus, line $L$ does not intersect all the diamonds. Any line that intersects $A 1$ before $A 4$ must cross the lower segment of point $b_{1}$. The line cannot intersect $A 2$ and $A 3$. A similar argument can be employed for any line intersecting $A 4$ before $A 1$. A line must cross the upper segment of point $b_{2}$; therefore, the line does not intersect $A 2$ and $A 3$. Fig. 7(b) shows that a diamond can be inserted between $A 2$ and $A 3$. The corresponding subsequence in $\Delta \theta[n]$ is 101 , and any number of diamonds can be inserted between $A 2$ and $A 3$. Similarly, we can show that $10^{*} 1$ is an impossible pattern. As the subsequence in $\Delta \theta[n]$ is $(-1) 0^{*}(-1)$, it is omitted from the proof.

- P2: $\Delta \theta[n]$ contains neither a subsequence $\cdots 010 \cdots$ nor $\cdots 0(-1) 0 \cdots$.

Proof: Fig. 7(c) illustrates that any line with a slope smaller or greater than the slope of line $L$ will intersect at most four diamonds. The corners in $A 1$ and $A 4$ where line $L$ intersects are not quantized to our lattice points. Thus, there is no line that can intersect all the diamonds. So sequence 010 is an impossible pattern for $\Delta \theta[n]$. Because a similar argument can be used for the sequence $0(-1) 0$, it is not included in the proof. The chain code can then be derived according to the

above two properties of $\Delta \theta[n]$.

Fig. 7. The following patterns are impossible in $\Delta \theta[n]$ for the chain code of a line: (a) two consecutive 1's; (b) subsequences 101 and $10(0)^{*} 1$; and (c) subsequence 010 . 
Case 1: There is a 0 in $\Delta \theta[n]$. Let the sequence be $\cdots 00^{*} 1 x$ and the corresponding sequence of the chain code be $\cdots \theta_{0} \theta_{0}^{*}\left(\theta_{0}+1\right) c(x)$. According to P1 and P2, the $x$ in $\Delta \theta[n]$ is neither 1 nor 0 ; hence, $x$ is -1 . The correspoinding chain code value $c(x)$ is therefore $\theta_{0}$. The corresponding piece of the chain code that contains a Dirac is

$$
\theta_{0} \theta_{0}^{*}\left(\theta_{0}+1\right) \theta_{0}
$$

A similar argument can be applied to a subequence of $\Delta \theta[n]: \cdots 00^{*}(-1) x$, as it obtains a Dirac at the corresponding piece of the chain code.

Case 2: There exists no 0 in $\Delta \theta[n]$. According to $\mathrm{P} 1$ and $\mathrm{P} 2$, $\Delta \theta[n]$ is $\cdots(-1) 1((-1) 1)^{*}$, and the corresponding piece of the chain code is

$$
\cdots \theta_{0}\left(\theta_{0}+1\right)\left(\theta_{0}\left(\theta_{0}+1\right)\right)^{*} \text {. }
$$

This piece of chain code contains many Diracs and the distance between two consecutive Diracs is at least 2. A similar argument to Case 2 can be applied when $\Delta \theta[n]$ is $\cdots 1(-1)(1(-1))^{*}$, which obtains a piece of the chain code

$$
\cdots \theta_{0}\left(\theta_{0}-1\right)\left(\theta_{0}\left(\theta_{0}-1\right)\right)^{*}
$$

The conclusion drawn from Cases 1 and 2 is that the chain code of an imaged line is a constant plus a Dirac sequence.

\section{ACKNOWLEDGMENT}

Wen-Liang Hwang would like to express his gratitude to Prof. S. Mallat, Prof. B. Torrésani, Prof. E. Bacry, and Prof. R. Carmona from whom he has learned much about modulus maxima and continuous wavelet transform. Also, all the authors would like to thank the Associated Editor for his invaluable assistance.

\section{REFERENCES}

[1] J. P. Antoine and R. Murenzi, "Two-dimensional directional wavelets and the scale-angle representation," Signal Process., vol. 52, no. 3, pp. 259-281, 1996.

[2] A. Arneodo, E. Bacry, and J. F. Muzy, "The thermodynamics of fractals revisited with wavelets," Phys. A, vol. 213, pp. 232-275, Jan. 1995.
[3] H. Asada and M. Brady, "The curvature primal sketch," IEEE Trans. Pattern Anal. Mach. Intell., vol. 8, no. 1, pp. 2-14, Jan. 1986.

[4] D. H. Ballard and C. M. Brown, Computer Vision. Englewood Cliffs, NJ: Prentice-Hall, 1982.

[5] R. Carmona, W. L. Hwang, and B. Torrésani, "Multiridge detection and time-frequency reconstruction," IEEE Trans. Signal Process., vol. 47, no. 2, pp. 480-492, Feb. 1999.

[6] E. Chassande-Mottin and P. Flandrin, "On the time-frequency detection of chirps," Appl. Comput. Harmonic Anal., vol. 6, pp. 252-281, 1999.

[7] N. Delprat, B. Escudié, P. Guillemain, R. Kronland-Martinet, P. Tchamitchian, and B. Torrésani, "Asymptotic wavelet and gabor analysis: Extraction of instantaneous frequencies," IEEE Trans. Inf. Theory, vol. 38, no. 2, pp. 644-664, Mar. 1992.

[8] R. C. Gonzalez and R. E. Woods, Digital Image Processing. Reading, MA: Addison-Wesley, 1993.

[9] A. Grossmann and J. Morlet, "Decomposition of hardy functions into square integrable wavelets of constant shape," SIAM J. Math. Anal., vol. 15, no. 4, pp. 723-736, Jul. 1984.

[10] M. Holschneider and P. Tchamitchian, Regularite Locale de la Fonction Non-Differentiable de Riemann (Lecture Notes in Mathematics), P. G. Lemarie, Ed. New York: Springer-Verlag, 1990, vol. 1438.

[11] W. L. Hwang, C. S. Lu, and P. C. Chung, "Shape from texture: Estimation of planar surface erientation through the ridge surfaces of continuous wavelet transform," IEEE Trans. Image Process., vol. 7, no. 5, pp. 773-780, May 1998.

[12] S. Mallat, A Wavelet Tour of Signal Processing. San Diego, CA: Academic, 1999.

[13] S. Mallat and S. Zhong, "Characterization of signals from multiscale edges," IEEE Trans. Pattern Anal. Mach. Intell., vol. 14, no. 7, pp. 710-732, Jul. 1992.

[14] S. Mallat and W. L. Hwang, "Singularity detection and processing with wavelets," IEEE Trans. Inf. Theory, vol. 38, no. 2, pp. 617-643, Mar. 1992.

[15] P. Guillemain, "Ridges associated to isolated frequential and temporal singularities," in Progress in Wavelet Analysis and Applications, Y. Meyer and S. Roques, Eds. Singapore: Editions Frontiers, 1992, pp. 277-284.

[16] D. Mar, Vision. San Francisco, CA: Freeman, 1982

[17] S. Jaffard, "Sur la dimension de Hausdorff des points singuliers d'une fonction," C. R. Acad. Sci., ser. I, vol. 308, pp. 79-81, 1989.

[18] _ - "Pointwise smoothness, two-microlocalization and wavelet coefficients," Publicacions Matemétiques, vol. 35, pp. 155-168, 1991.

[19] S. Jaffard and Y. Meyer, "Wavelet methods for pointwise regularity and local oscillations of functions," Amer. Math. Soc., vol. 123, no. 587, Sep. 1996.

[20] F. Mokhtarian and A. Mackworth, "A theory of multiscale, curvaturebased shape representation for planar curves," IEEE Trans. Pattern Anal. Mach. Intell., vol. 14, no. 8, pp. 789-805, Aug. 1992.

[21] J. M. Steele and W. L. Steiger, "Algorithm and complexity for least median of squares regression," Discr. Appl. Math., vol. 14, pp. 93-100, 1986.

[22] P. Tchamitchian and B. Torrésani, "Ridge and skeleton extraction from the wavelet transform," in Wavelets and Their Applications. Boston, MA: Jones and Bartlett, 1992, pp. 123-151.

[23] A. Witkin, "Scale space filtering," in Proc. Int. Joint. Conf. Artificial Intelligence, Espoo, Finland, Jun. 1983. 